\title{
Reforma psiquiátrica: Percursos, realidades e desafios
}

\author{
Psychiatric reform: Paths, realities and challenges \\ Reforma psiquiátrica: Caminos, realidades y desafios
}

\section{Resumo}

O artigo objetiva apresentar reflexões sobre a Reforma Psiquiátrica brasileira, sua trajetória histórica e seus desafios atuais, incorporando a compreensão de que a mesma pode ser conceituada como um processo civilizador, de respeito aos direitos de cidadania da pessoa em sofrimento psíquico e não apenas como uma mudança de estrutura ou de instituição de saúde. Trata-se de um artigo de reflexão, com sessões que abordam aspectos importantes neste contexto, como a reabilitação psicossocial, a redução de danos e a promoção do cuidado à saúde mental. Diante de um cenário em que os avanços conquistados nas três décadas da Reforma Psiquiátrica no Brasil parecem estar em processo de retrocesso, apresentando uma tendência de (re)manicomialização do cuidado em saúde mental, enfatiza-se que é necessária a sensibilização coletiva, promovendo uma assistência em saúde mental mais ampliada e humanizada, combatendo estigmas e preconceitos e reivindicando conquistas da reforma, por meio de contínuos movimentos sociais.

Palavras-chave: Saúde pública; Saúde mental; Cuidados de saúde.

\begin{abstract}
The article aims to present reflections on the Brazilian Psychiatric Reform, its historical trajectory and its current challenges, incorporating the understanding that it can be conceptualized as a civilizing process, respecting the citizenship rights of people in mental suffering and not just as a change of structure or health institution. This is a reflection article, with sessions that address important aspects in this context, such as psychosocial rehabilitation, harm reduction and the promotion of mental health care. Faced with a scenario in which the advances achieved in the three decades of the Psychiatric Reform in Brazil seem to be in a process of setback, showing a trend of (re)manicomialization of mental health care, it is emphasized that collective awareness is necessary, promoting a more expanded and humanized mental health care, fighting stigma and prejudice and claiming the achievements of the reform, through continuous social movements.
\end{abstract}

Keywords: Public health; Mental health; Health care.

\section{Resumen}

El artículo tiene como objetivo presentar reflexiones sobre la Reforma Psiquiátrica Brasileña, su trayectoria histórica y sus desafíos actuales, incorporando el entendimiento de que puede ser conceptualizada como un proceso civilizador, respetando los derechos de ciudadanía de las personas en sufrimiento mental y no solo como un cambio de estructura. 
o institución de salud. Este es un artículo de reflexión, con sesiones que abordan aspectos importantes en este contexto, como la rehabilitación psicosocial, la reducción de daños y la promoción de la atención en salud mental. Ante un escenario en el que los avances logrados en las tres décadas de la Reforma Psiquiátrica en Brasil parecen estar en un proceso de retroceso, mostrando una tendencia de (re) manicomialización de la atención en salud mental, se enfatiza que la conciencia colectiva es necesaria, promoviendo una atención de la salud mental más ampliada y humanizada, combatiendo el estigma y los prejuicios y reivindicando los logros de la reforma, a través de continuos movimientos sociales.

Palabras clave: Salud pública; Salud mental; Cuidados de la salud.

\section{Introdução}

A Reforma Psiquiátrica brasileira teve início na segunda metade da década de 70, porém, o processo se intensificou nos anos 90 por meio de movimentos sociais, com destaque aos trabalhadores da saúde mental, que lutaram por uma transformação na assistência e criaram novos paradigmas (Yasui, 2006; Tenório, 2002). Ela se caracteriza por uma ruptura nos modelos hegemônicos, para que novas formas de cuidado na saúde mental sejam criadas: com ética, liberdade, autonomia e valor social, outrossim, manifesta a ressignificação e a construção de um novo lugar para a loucura (Yasui, 2006; Amarante \& Nunes, 2018).

Contextualizando a Reforma Psiquiátrica em um percurso histórico, no ano de 1980, com a crise financeira do Instituto Nacional de Assistência Médica da Previdência (INAMPS), houve a revelação que o número de leitos psiquiátricos aumentou de forma acentuada durante o regime dos governos militares, nesse cenário, grande parte da população tinha acesso somente a grandes e semiabandonados asilos públicos. A assistência psiquiátrica se limitava principalmente a internações hospitalares, característica do modelo biomédico, hospitalocêntrico e mercantilizado, constituindo a "indústria da loucura" (Delgado, 2019).

Nesse período, muitas denúncias foram feitas, relacionadas a fraude no sistema de financiamento dos serviços, do abandono, da violência e dos maus-tratos que os pacientes internados nos grandes asilos (hospícios/manicômios) de todo país, eram submetidos. Em 1978, surge um movimento de grande repercussão para reforma, o Movimento dos Trabalhadores em Saúde Mental (MTSM), que lutavam por reformas trabalhistas e por uma assistência psiquiátrica mais humanizada. Posteriormente, usuários, familiares e diversos atores sociais também ingressaram, constituindo o movimento da "Luta antimanicomial: por uma sociedade sem manicômios", sendo instituído o dia 18 de maio como o dia da luta antimanicomial (Tenório, 2002).

Paralelo a Reforma Psiquiátrica, estava a Reforma Sanitária, a qual reivindicava pela reformulação da política nacional de saúde, e entre os seus objetivos, o de ampliar o acesso da população à assistência em saúde no geral (Tenório, 2002). A partir daí a Reforma Psiquiátrica seguiu duas vertentes autolimitadas: a humanização dos asilos públicos (modelo das comunidades terapêuticas) e a ambulatorização da saúde mental, tendo como perspectiva os direitos de cidadania (Delgado, 2019).

Alguns eventos foram essenciais para as transformações permeadas pela Reforma Psiquiátrica, a I Conferência Nacional de Saúde Mental e o II Encontro Nacional dos Trabalhadores em Saúde Mental, ambos em 1987. A partir disso, deuse início a uma trajetória de desconstrução das instituições tradicionais (asilos/hospícios/manicômios) e na reconstrução das formas de lidar com o "louco" e com a "loucura", a chamada desinstitucionalização. Destaca-se também a constituição de 1988, sendo fundamental para os avanços da reforma e para as políticas de saúde como um todo (Delgado, 2019).

Dentre os principais marcos desses movimentos e eventos, estão: a redução dos leitos de hospital especializado, criação de serviços na comunidade, ampliação do acesso ao tratamento, fortalecimento do atendimento na atenção primária e a intersetorialidade (Delgado, 2019). Incorporando um fortalecimento entre os usuários do serviço (como passam a ser chamados) e familiares, “[...] que, seja na relação direta com os cuidadores, seja através de suas organizações, passam a ser 
verdadeiros agentes críticos e impulsionadores do processo" (Tenório, 2002, p. 35).

Em 1989, iniciou-se uma busca por uma revisão legislativa, por meio de um projeto de lei n. 3.657/89, com três artigos principais: em que a construção ou contratação de novos hospitais psiquiátricos seriam impedidos; direcionamento dos recursos públicos para a criação de dispositivos não-manicomiais; e a obrigatoriedade da comunicação das internações compulsórias à autoridades judiciárias, que deveriam emitir parecer jurídico sobre a legalidade da internação. Anos depois, após muitos percalços em sua tramitação, foi promulgada a lei 10.216 de 6 de abril de 2001, conhecida como a Lei Paulo Delgado (Tenório, 2002).

A lei 10.216/2001 dispõe sobre a proteção e os direitos das pessoas portadoras de transtornos mentais e redireciona o modelo assistencial em saúde mental (Brasil, 2001). Diante disso, fica evidenciado que não se trata de aperfeiçoar ambulatórios e/ou asilos, mas sim, criar novos dispositivos e formas de cuidado, alicerçados em dispositivos abertos e de natureza comunitária e territoriais, conforme os preceitos da reforma psiquiátrica (Tenório, 2002).

Nesse contexto, surgem novos dispositivos de saúde mental, sendo o principal o Centro de Atenção Psicossocial (CAPS). O primeiro CAPS no Brasil foi inaugurado no ano de 1986, em São Paulo, conhecido como Centro de Atenção Psicossocial Professor Luiz da Rocha Cerqueira. Inicialmente, os CAPS foram criados pela portaria SAS/MS n. 224/1992, e posteriormente foram regulamentados pela portaria MS/GM n. 336/2002, sendo ampliado seu funcionamento e sua complexidade reconhecida (Brasil, 2004a). Esse serviço tem como objetivo, prestar atendimento às pessoas com transtornos mentais graves e persistentes e aquelas com necessidades decorrentes do uso de álcool e outras drogas, atuando de forma articulada com os outros pontos de atenção da rede de saúde e das demais redes (Brasil, 2004a; Brasil, 2013).

Outro marco importante para saúde mental, foi a instituição da Rede de Atenção Psicossocial (RAPS), por meio da portaria MS/GM n. 3.088/2011, a qual teve como objetivo ampliar e articular os pontos de atenção à saúde para as pessoas em sofrimento psíquico ou transtorno mental e com necessidades decorrentes do uso de álcool e outras drogas, nos diferentes níveis de complexidade. Abrange-se os seguintes componentes: a atenção básica, atenção psicossocial especializada, atenção de urgência e emergência, atenção residencial de caráter transitório, atenção hospitalar, estratégias de desinstitucionalização e reabilitação psicossocial (Brasil, 2011; Brasil, 2013).

Dessa maneira, o cuidado em saúde deve ser produzido com a articulação e corresponsabilidade entre serviços e sujeitos, em seus diferentes enfoques, disciplinas e integração de diversas abordagens, objetivando o manejo eficaz do trabalho em saúde (Brasil, 2009). Nessa visão de clínica ampliada, as articulações intersetoriais são cruciais para combater diferenças, incluir os sujeitos e fortalecer a equidade e a cidadania (Amarante \& Torre, 2018).

O território em que ocorre esse cuidado, vai além de um espaço geográfico, a "territorialidade estaria intimamente ligada ao modo como as pessoas utilizam a terra, como elas próprias se organizam no espaço e como elas dão significado ao lugar" (Monken et al., 2008, p. 5). O território em saúde mental é, então, amplo e considerado um espaço em que o indivíduo, família, comunidade e os serviços se relacionam e fazem parte do processo de cuidar das pessoas em sofrimento psíquico. Nesse contexto, os serviços do território possuem suas responsabilidades na assistência à saúde mental, e devem se articular visando a qualidade de vida e saúde da população.

No entanto, desde 2016 as políticas de saúde no país vêm sofrendo um retrocesso. Dentre as ações desse declínio, está uma medida provisória, que se tornou a Emenda Constitucional 95/2016, a qual congelou por 20 anos os recursos que deveriam ser destinados ao Sistema Único de Saúde (SUS) e outras políticas sociais. No campo da saúde mental, os principais retrocessos foram na estrutura da RAPS e na ampliação do financiamento de internações psiquiátricas e de comunidades terapêuticas. Em contrapartida, a implantação e recursos destinados aos CAPS, foram diminuídos (Cruz, Gonçalves, \& Delgado, 2020). Medidas essas, contrarreforma psiquiátrica fortalecem um modelo desterritorializado.

Outro impacto importante, foi a modificação da Política Nacional de Atenção Básica (PNAB), pela portaria n. 
2.436/2017, entre as alterações, está a retirada da palavra "democrática" do texto atual e a desaparição da "humanização" como princípio. Também houve uma lacuna com relação ao número mínimo de Agentes Comunitários de Saúde (ACS) por equipe, sem definição do número atual, passando a ser 1 ACS/equipe, sendo que na edição anterior da PNAB, eram 4 ACS/equipe (Melo, Mendonça, Oliveira, \& Andrade, 2018). Tais medidas atingem a atenção psicossocial, no sentido que uma atenção primária com um número defasado de ACS's, impacta no cuidado de base comunitária e na integralização do cuidado (Cruz, Gonçalves, \& Delgado, 2020).

Diante esse cenário, é necessário o envolvimento da sociedade na defesa dos direitos das pessoas em sofrimento psíquico e na continuidade do movimento da luta antimanicomial, fortalecendo os princípios e estratégias da Reforma Psiquiátrica. Entre eles, o desenvolvimento do trabalho em rede, que por meio da articulação entre os serviços e vivências interdisciplinares de diferentes categorias profissionais, promovem a humanização e o cuidado integral em saúde mental (Nogueira \& Campos, 2016). Com destaque aos CAPS, que possui papel fundamental nesse trabalho e no apoio matricial.

Os CAPS devem trabalhar de forma integrada com o serviços de saúde do seu território, interagindo com as equipes, realizando levantamentos sobre os problemas relacionados a saúde mental da população, buscando em conjunto, estratégias para atender essas necessidades e oferecer o matriciamento, por meio de orientação, supervisão, visitas domiciliares e suporte para situações complexas, quando necessário e/ou solicitado, em especial para atenção primária, porta de entrada do SUS (Brasil, 2004a).

No entanto, é preciso compreender que não é somente o CAPS o responsável por essa assistência, é necessário o envolvimento e participação de outros setores da sociedade tais como, educação, assistência social, cultura, entre outros. A integralidade em saúde e uma adequada articulação entre os serviços promovem qualidade de vida e respeito a autonomia e direitos da pessoa em sofrimento psíquico.

Outro ponto, é a notoriedade do Projeto Terapêutico Singular (PTS), um conjunto de estratégias terapêuticas, construído de forma coletiva e articulada (usuário, família, equipe interdisciplinar) para um indivíduo/família/grupo. Dessa forma, o PTS é resultado de discussões de reuniões de equipe, no intuito de atender/facilitar demandas e necessidades que vão além do diagnóstico clínico e da medicalização no tratamento do usuário. Busca-se a singularidade, a minimização das diferenças e o fortalecimento das potencialidades, respeitando o protagonismo e autonomia do sujeito/família nesse processo (Brasil, 2009).

Continuamente é preciso sensibilizar a sociedade, em seus diversos segmentos (usuário, família, profissionais), quanto aos direitos das pessoas em sofrimento psíquico e em situações de vulnerabilidade, por meio de debates, participação social, arte-cultura, inclusão pelo trabalho e no diálogo de políticas públicas com os movimentos sociais. Contribuições importantes para o movimento da luta antimanicomial e no processo de transformação no campo da saúde mental (Amarante \& Torre, 2018).

A qualificação e a capacitação dos profissionais também são essenciais para o acolhimento da pessoa em sofrimento psíquico e para prática do cuidado integral em saúde mental. Isso pode ser realizado por meio de diferentes estratégias, dentre elas, a adequação dos currículos de graduação, fortalecendo os preceitos da Reforma Psiquiátrica e preparando o acadêmico para atuação no território, com enfoque no trabalho interdisciplinar (Silva, Esperidião, Cavalcante, Souza \& Silva, 2013). Ou ainda, promovendo o aperfeiçoamento em saúde mental, que pode ser incentivado pela gestão pública, abrangendo distintas categorias profissionais (enfermagem, nutrição, fisioterapia, farmácia, serviço social, etc.), com atuação nos diferentes níveis de complexidade, possibilitando a discussão, aprendizagem e compartilhamento das vivências e saberes (Nogueira \& Campos, 2016).

Ainda, é preciso priorizar a assistência por meio das Redes de Atenção à Saúde (RAS), superando o modelo "piramidal", que prejudica o fluxo de atendimento em saúde mental, pois, o trabalho em forma de encaminhamento, referência 
e contra referência, além de enrijecer o processo de trabalho, também gera a ideia de isenção de responsabilidade do serviço quando encaminha o usuário para outro dispositivo. Essa forma de atendimento, além de fragmentada, gera frustração no usuário, muitas vezes agravando o seu caso. Já a RAS, tem como objetivo promover a saúde com articulações multidisciplinares, de forma contínua, integral e humanizada, em todos os níveis de complexidade, visando uma assistência à saúde eficaz (Guimarães, 2016).

O tratamento das pessoas em sofrimento psíquico deve ser realizado de forma acolhedora e humanizada, livre de qualquer tipo de preconceito, tendo em vista a reinserção social do indivíduo, na sua família e comunidade, empregando tecnologias leves para acolhimento e escuta qualificada. Também é essencial a criação de vínculo terapêutico, a utilização de Práticas Integrativas e Complementares, entre outras estratégias, estabelecidas pela Política Nacional de Humanização e outras políticas públicas em saúde mental (Brasil, 2004a; Brasil, 2009; Brasil, 2013). Além disso, outros aspectos a serem discutidos a seguir precisam ser considerados e se tornam importantes ao contexto apresentado.

\section{Metodologia}

Trata-se de um artigo de reflexão, fundamentado na discussão teórica, crítica e descritiva sobre a Reforma Psiquiátrica brasileira, considerando seus percursos, realidades e desafios atuais. São incorporadas sessões que abordam aspectos importantes nesse contexto, como a reabilitação psicossocial, a redução de danos e a promoção do cuidado à saúde mental.

\subsection{Reabilitação psicossocial: variáveis e eixos da vida real}

As mudanças oriundas da Reforma Psiquiátrica vem acontecendo no modo como se dá o cuidado em saúde mental. Um exemplo pode ser percebido na concepção de saúde mental de base comunitária que traz consigo diversos atores e os mais diversificados serviços, e não apenas o usuário e o serviço psiquiátrico; mas sua família, a Atenção Primária em Saúde (APS), o convívio social, entre outros (Brasil, 2017; Saraceno, 2001; Cruz, Gonçalves, \& Delgado, 2019).

Vivemos em três cenários distintos considerados como eixos que se completam: casa, trabalho e rede social (mercado, campo de troca). Nesses cenários de vida há pessoas com mais habilidade contratual para negociação do que outros. Mas há também quem seja desabilitado por não ter poder contratual, e é aí que entra o processo de reabilitação (Saraceno, 1996).

Uma pessoa que está desabilitada devido ao transtorno mental pode ter sua vida desajustada e isso vai interferir no modo como ela vive em sociedade. Somado aos desajustes causados pelo transtorno mental, o hospital psiquiátrico (manicômios) induz às pessoas ditas como "loucas" ao distanciamento social causado pelo afastamento da família, da cidade, do trabalho, do lazer, privando o sujeito do direito à cidadania (Amarante, \& Nunes, 2018).

A Reabilitação Psicossocial (RP) é um processo de reconstrução que busca impulsionar as habilidades da pessoa com transtorno mental, ou seja, que suas potencialidades sejam reconhecidas e usadas nos cenários do cotidiano para alavancar sua autonomia, a sua reinserção na sociedade, e com isso resgatar o protagonismo perdido, aumentar seu poder contratual, de modo a viver melhor em seu meio no exercício de sua cidadania plenamente (Saraceno, 1996; 2001). Enfatize-se que é no setting do cotidiano que as práticas da vida se constituem e se faz reabilitação (Kantorski, Cardano, Borges, \& Machado, 2021).

Para que o processo da reabilitação seja colocado em prática é preciso que as pessoas envolvidas (indivíduo com transtorno mental, a família, a comunidade, os profissionais de saúde) e os espaços sociais (a cidade, o comércio, o serviço de saúde) teçam as relações e estejam em constante negociação de contratualidade (Saraceno, 2001). Se a relação contratual está centrada apenas nos serviços de saúde, a inserção e autonomia desses sujeitos em reabilitação fica comprometida (Acebal, Barbosa, Domingos, Bocchi, \& Paiva, 2020; Cortes, \& Barros, 2017).

Outro comprometimento para a reabilitação psicossocial no Brasil, têm acontecido a partir de meados do ano de 2016 até os dias atuais e se traduz em processo acelerado de desmonte dos avanços adquiridos pela Reforma Psiquiátrica (Delgado, 
2019). Inúmeros documentos normativos têm sido publicados e revelam a tendência do retrocesso ao modelo manicomial muito exercido antes da reforma (Brasil, 2019a).

Todo o desmonte causado por práticas e cortes advindos dessas normativas afeta diretamente os serviços de saúde mental brasileiros. Isso pode levar à reflexão do quanto todos os setores, que já apresentavam problemas estruturais, foram e serão afetados por atos de contrarreforma (Cruz, Gonçalves, \& Delgado, 2019). Nesse sentido, é preciso resistir e lutar para que todas as conquistas no campo da atenção psicossocial, pautadas no atendimento por meio da rede de serviços de base comunitária e de orçamento para estes serviços, sejam prioridade nas políticas públicas em saúde mental.

Ademais, que novas abordagens possam ser apreendidas e incrementadas, oriundas de experiências exitosas, de outros países que trabalham mais para fora (na cidade, nas ruas, no mercado e não apenas centralizada no serviço de saúde), de modo que propiciem mais empoderamento e sejam pautadas na crença de que as pessoas são capazes de traçar seus objetivos, apesar de serem afetadas por um declínio mental.

\subsection{A construção de saberes e práticas relacionadas ao álcool e drogas sob a perspectiva da redução de danos}

Ao considerar os modelos de atenção à saúde direcionado às pessoas que fazem uso abusivo de Substâncias Psicoativas (PSA), ou seja, de álcool e/ou outras drogas lícitas ou ilícitas, torna-se necessário problematizar sobre a adequação dos saberes e práticas de cuidados implementados por profissionais de saúde e outros, a luz das políticas públicas, com vistas a minimizar os danos, com a redução das vulnerabilidades e a promoção e o fortalecimento dos fatores de proteção, para ao mesmo tempo, melhorar a qualidade de vida dessas pessoas.

Considera-se que a construção e a implementação de políticas públicas definirão a reprodução de um modelo de atenção à saúde, assim como para a orientação dos saberes e práticas de trabalho dos profissionais que atuam nos serviços de atenção à saúde mental. Nesse sentido, destaca-se o quanto as políticas públicas relacionadas ao consumo abusivo de álcool e outras drogas, são determinantes para além da organização da rede de atenção integral, como também para o trabalho dos profissionais de saúde que assistem as pessoas que fazem o uso e abuso de PSA.

A Política de Atenção para Usuários de Álcool e Outras Drogas, foi redefinida e ampliada sendo atualmente regulamentada pela Portaria $\mathrm{N}^{\circ}$ 2.197, de outubro de 2004 (Brasil, 2004b). Destaca-se que essa política tem nos princípios do SUS e da Reforma Psiquiátrica os seus eixos centrais para sua implementação considerando as necessidades e especificidades para a atenção do usuário de álcool e outras drogas (Santos \& Oliveira, 2013). Para o funcionamento dessa política, foram definidos os seguintes componentes: o componente da atenção primária; da atenção nos CAPS-AD, ambulatórios e outras unidades extra-hospitalares especializadas; da atenção hospitalar de referência; e o componente da rede de suporte social, como as associações de ajuda e entidades da sociedade civil que são complementares à rede de serviços que são disponibilizados pelo SUS (Brasil, 2004b).

Destaca-se também o quanto é essencial o apoio de uma equipe multidisciplinar de saúde, desde o serviço de atenção primária, ou da atenção nos CAPS-AD (Centros de Atenção Psicossocial Álcool e drogas), ambulatórios ou unidades extrahospitalares especializadas ou atenção hospitalar de referência. Ressalta-se, ainda, a importância para um cuidar qualificado, não somente para o indivíduo em tratamento, mas também para sua família. Nesta perspectiva, a família também deve ser inserida no plano de cuidado, pois também está adoecida e fragilizada, e precisa de apoio, para posteriormente conseguir apoiar e contribuir para a reinserção social do seu ente querido. Ser inserido novamente na família e ser "aceito" na sociedade, resgatando o seu papel social é muito mais do que pensar somente em saúde, representa a promoção da cidadania e o resgate da dignidade humana.

Quanto a legislação brasileira sobre drogas, a Política Nacional sobre Drogas, foi aprovada pelo Decreto $\mathrm{n}^{\circ}$. 9.761/2019, revogou inteiramente o Decreto $\mathrm{n}^{\circ}$. 4.345, de 26 de agosto de 2002 (Brasil, 2019b). A reflexão crítica refere-se ao 
fato que essa nova política não faz menção às práticas de redução de danos, enquanto ressalta a promoção e a manutenção da abstinência.

A abordagem de redução de danos, antes considerada na esfera das políticas relacionadas ao consumo abusivo de álcool e outras drogas, orienta para modelos de práticas e intervenções com o intuito de minimizar os danos à saúde, assim como para os prejuízos sociais e econômicos relacionados ao abuso de drogas, sem necessariamente proibi-las. Nesse sentido, Dias, Lopes e Marangoni (2020) referem que a redução de danos é uma estratégia utilizada em vários programas de saúde no Brasil.

Compreende-se que a partir desse modelo de redução de danos, que não é direcionado para o proibicionismo do consumo das PSA com autoritarismo nas intervenções, mas que se volta para indivíduo, considerando a sua inserção em uma família/sociedade, para uma atenção humanizada, pautado no diálogo com uma escuta atenta e solidária, considerando as suas necessidades e em práticas e saberes com vistas a agregar dignidade e qualidade de vida à essas pessoas que muito já sofrem com a dependência química.

Nesse sentido, entende-se que o modelo de redução de danos é importante para orientar as práticas dos profissionais em diferentes cenários de atenção à saúde, devendo ocorrer desde a APS até os demais níveis de atenção, assim como considerar a comunidade e os territórios de responsabilidade dos serviços de saúde, como as Unidades Básicas, os CAPS e também articular com outras redes de serviços de saúde e os serviços sociais.

Santos, Aguiar e Braga (2020) contribuem com essa discussão ao afirmarem que a redução de danos visa oferecer um suporte necessário a esses indivíduos (que abusam das PSA), pois possibilita o escutar sobre as suas necessidades de maneira não preconceituosa, respeitando as suas escolhas e realizando as ações que são possíveis para um momento oportuno, ressaltando o compromisso da política pública como serviço de apoio a este indivíduo com o intuito de reabilitá-lo para a vida em sociedade.

Torna-se também fundamental repensar sobre o significado na atualidade da redução de danos, e nesse aspecto, assume-se uma concepção ampla, pois considera os direitos individuais e sociais, com o objetivo de minimizar danos sociais, culturais, econômicos, afetivos e outros, além dos danos da saúde do indivíduo que abusa das PSA, viabilizando o acesso aos serviços com profissionais qualificados, de modo a multiplicar as possibilidades de enfrentamento às vulnerabilidades relacionadas com o abuso das SPA (Santos, Aguiar, \& Braga, 2020).

Apesar da importância de todos esses aspectos apresentados anteriormente, a nova Política Nacional sobre Drogas trouxe mudanças negativas para o modelo de cuidado, enfraquecendo as ações que são fundamentais para a implementação da Política de Redução de Danos, como por exemplo, o estímulo às práticas que valorizam a abstinência total e um aumento do financiamento público em Comunidades Terapêuticas, tidas como características do modelo proibicionista. Além de que, ao restringir as práticas e ações essencialmente na abstinência, é anulado qualquer possibilidade de promoção da saúde para as pessoas que não conseguem ou não desejam tal situação (Dias, Lopes, \& Marangoni, 2020).

Considera-se que essas mudanças caracterizam um retrocesso para o modelo de atenção orientado pela redução de danos, situação que fragiliza as ações e práticas de cuidados direcionados à essas pessoas que abusam das PSA, pois não consideram as vulnerabilidades e iniquidades sociais, assim como não aceitam a participação desse indivíduo e sua família na tomada de decisões quanto ao tratamento terapêutico oportuno, entre outros. Nesse aspecto, tem-se a convicção da importância da redução de danos direcionadas ao cuidado às pessoas que abusam das PSA, sendo que a efetividade do cuidado e das práticas, são aspectos de inclusão e participação social desse indivíduo, muito além da abstinência, que não é o foco desse modelo.

As estratégias de redução de danos colaboram para a melhoria das condições de vida e saúde das pessoas e suas famílias, fortalece os vínculos, gera oportunidades de inclusão social além de oportunizar o diálogo, em que esse indivíduo, 
repensa sua relação com o abuso de drogas e passa a se ver como um protagonista da sua vida e das suas escolhas, sempre tendo o suporte de uma equipe multiprofissional qualificada, independentemente de quantas recaídas tiver ou do tempo que necessitar desse cuidado.

\subsection{O cuidado como desafio para o pensar e o fazer nas práticas de saúde}

Também faz-se necessário refletir acerca das práticas de saúde contemporâneas e os desafios existentes na promoção do cuidado. Apesar do significativo desenvolvimento científico e tecnológico, as práticas de saúde têm demonstrado sérias limitações, recebendo críticas sobre a visão segmentada; por não observar a totalidade do usuário e o contexto em que está inserido; por apresentar uma abordagem muito centrada na patologia; pela superficialidade da relação e interação entre profissionais e usuários e entre os demais profissionais da equipe; e, sobretudo, o frágil compromisso com o bem-estar dos usuários (Ayres, 2009).

O fazer em saúde tem se reconstruído a partir de estratégias de humanização e integralidade do cuidado em saúde, por meio do acolhimento, vínculo e responsabilização na organização da assistência à saúde. O cuidar nas práticas de saúde institui-se no desenvolvimento de atitudes e espaços de encontro intersubjetivo, no exercício de uma sabedoria prática para a saúde, amparados na tecnologia, mas sem restringir-se a ela a ação em saúde (Ayres, 2004).

Especificamente no campo da saúde mental, o movimento sanitário e a Reforma Psiquiátrica defenderam a reordenação do entendimento da saúde, como supracitado. A proposta deste novo modo de cuidar traz o sujeito, sua família e a comunidade como os elementos centrais do processo terapêutico em saúde mental em oposição à centralidade na doença e no manicômio do paradigma existente até então (Kinker, Moreira, \& Bertuol, 2018). A Política Nacional de Saúde Mental (PNSM) redirecionou os modos de produção de cuidado em saúde mental, ao preconizar a realização do tratamento em serviços de base territorial, como os CAPS, e na APS, por meio da equipe de saúde da família, entre outros, despersuadindo o isolamento do indivíduo do seio familiar e comunitário (Ferreira, Sampaio, Oliveira, \& Gomes, 2019).

Outro aspecto é de que as ações do profissional enfermeiro no âmbito da saúde mental estão relacionadas, em especial, às ações interdisciplinares sob a égide psicossocial. O enfermeiro é a "porta de entrada" para o cuidado, facilitador e integrador de ações por possuir mais contato com o usuário (Almeida, 2020). Nesta perspectiva do cuidado, considera-se de extrema importância a discussão de pensar a prática clínica voltada à saúde mental como um espaço onde as narrativas dos sujeitos sejam produzidas e valorizadas, permitindo a abertura para o reconhecimento dos Determinantes Sociais da Saúde (DSS) (Pellegrini, 2021) e a construção de saberes e práticas psicossociais às populações em situação de vulnerabilidade.

O estudo sobre as relações entre determinantes sociais e saúde mental proporciona um melhor conhecimento de como as intervenções devem ser feitas, buscando minimizar as iniquidades de saúde e contribuir na promoção de estilos de vida saudáveis; redução de fatores de risco para as perturbações mentais e comportamentais, e na melhoria da saúde mental das populações (Alves \& Rodrigues, 2010).

Considerando, portanto, as circunstâncias nas quais as pessoas nascem, crescem, vivem, trabalham e envelhecem, reforça-se a importância da intersetorialidade no cuidado em saúde, permitindo uma assistência mais ampliada, com interação afetiva e terapêutica que minimize os danos da descontinuidade do cuidado. Além da necessidade de implementação e fortalecimento de programas que se destinem à promoção da saúde mental e a redução das problemáticas de saúde mental e seus determinantes.

\section{Considerações Finais}

Diante do que foi apresentado enfatiza-se que a atual existência de um movimento antirreformista da nova/velha política de saúde mental no Brasil, reapresenta métodos e práticas que haviam sido abolidas. Os avanços conquistados nas três 
décadas da Reforma Psiquiátrica brasileira parecem estar em processo de retrocesso. O que vemos é a tendência de (re)manicomialização do cuidado em saúde mental no país, haja vista que a nova proposta da política de saúde mental tem a centralidade do cuidado na internação em hospitais psiquiátricos e em comunidades terapêuticas, salientando o crescente financiamento destes dispositivos, atendendo a indústria da doença e da loucura e a possibilidade comercial que isso representa.

Este cenário potencializa os desafios inerentes na assistência de pessoas em sofrimento psíquico. E nesse sentido, questionamos: o que podemos fazer enquanto profissionais e comunidade acadêmica para lutarmos por um cuidado em liberdade desta população? Como defender tudo que foi construído nas últimas três décadas da Reforma Psiquiátrica?

É necessário cada vez mais multiplicarmos informações, incentivando e sensibilizando a comunidade acadêmica, indivíduos e famílias, para uma assistência em saúde mental humanizada, combatendo estigmas e preconceitos e fazendo valer todas reivindicações e conquistas da reforma, por meio de contínuos movimentos sociais. Porque a luta antimanicomial continua, e mais do que nunca, fazemos parte dela!

\section{Referências}

Acebal, J. S., Barbosa, G. C., Domingos, T. S., Bocchi, S. C. M., \& Paiva, A. T. U. (2020). O habitar na reabilitação psicossocial: análise entre dois Serviços Residenciais Terapêuticos. Saúde em Debate, 44(127), 1120-1133.

Almeida, J. C. P., Barbosa, C. A., Almeida, L. Y., Oliveira, J. L., \& Souza, J. de (2020). Ações de saúde mental e o trabalho do enfermeiro. Revista Brasileira de Enfermagem, 73(1), e20190376.

Alves, A. A. M., \& Rodrigues, N. F. R. (2010). Determinantes sociais e econômicos da saúde mental. Revista Portuguesa de Saúde Pública, $28(2)$, 127-131.

Amarante, P., \& Torre, E. H. G. (2018). De volta à cidade, sr. cidadão! - reforma psiquiátrica e participação social: do isolamento institucional ao movimento antimanicomial. Revista de Administração Pública, 52(6), 1090-1107.

Amarante, P., \& Nunes, M. de O. (2018). A reforma psiquiátrica no SUS e a luta por uma sociedade sem manicômios. Ciência \& Saúde Coletiva, 23(6), 2067-2074.

Ayres, J. R. C. M. (2004). Care and reconstruction in healthcare practices, Interface - Comunicação, Saúde, Educação, 8(14), 73-92.

Ayres, J. R. C. M. (2009). Organização das ações de atenção à saúde: modelos e práticas. Saúde e Sociedade, 18(2), 11-23.

Brasil (2001). Lei no 10.216 de 6 de abril de 2001. Dispõe sobre a proteção e os direitos das pessoas portadoras de transtornos mentais e redireciona o modelo assistencial em saúde mental. Brasília, DF.

Brasil (2004a). Ministério da Saúde. Saúde Mental no SUS: Os Centros de Atenção Psicossocial.

Brasil (2004b). Ministério da Saúde. Portaria $n^{o} 2.197$, de 14 de outubro de 2004. Redefine e amplia a atenção integral para usuários de álcool e outras drogas, no âmbito do sistema único de saúde - sus, e dá outras providências. Brasília, DF.

Brasil (2009). Ministério da Saúde. Política Nacional de Humanização da Atenção e Gestão do SUS: Clínica ampliada e compartilhada.

Brasil (2011). Portaria $n^{o}$ 3.088, de 23 de dezembro de 2011. Institui a Rede de Atenção Psicossocial para pessoas com sofrimento ou transtorno mental e com necessidades decorrentes do uso de crack, álcool e outras drogas, no âmbito do Sistema Único de Saúde (SUS).

Brasil (2013). Ministério da Saúde. Cadernos de Atenção Básica: Saúde Mental. 176 p.

Brasil (2017). Ministério da Saúde. Portaria $n^{o} 3.588$, de 21 de dezembro de 2017. Institui sobre a Rede de Atenção Psicossocial, e dá outras providências no âmbito do Sistema Único de Saúde (SUS). Brasília, DF.

Brasil (2019a). Ministério da Saúde. Secretaria de Atenção à Saúde. Departamento de Ações Programáticas Estratégicas. Coordenação-Geral de Saúde Mental, Álcool e Outras Drogas. Nota Técnica $n^{\circ} 11 / 2019$, de 4 de fevereiro de 2019.

Brasil (2019b). Decreto $n^{\circ} 9.761$ de 11 de abril de 2019. Aprova a Política Nacional sobre Drogas.

Cortes, H. M., \& Barros, S. (2017). Reabilitação psicossocial de moradores de um serviço residencial terapêutico. Journal of Nursing and Health, 7(2), 14863.

Cruz, N. F. O., Gonçalves, R. B., \& Delgado, P. G. G. (2020). Retrocesso da reforma psiquiátrica: o desmonte da política nacional de saúde mental brasileira de 2016 a 2019. Trabalho, educação e saúde, 18(3), e00285117.

Delgado, P. G. (2019). Reforma psiquiátrica: estratégias para resistir ao desmonte. Trabalho, educação e saúde, 17(2), 1-4. 
Research, Society and Development, v. 10, n. 7, e29610716556, 2021

(CC BY 4.0) | ISSN 2525-3409 | DOI: http://dx.doi.org/10.33448/rsd-v10i7.16556

Dias, M. A. S.; Lopes, L. O., \& Marangoni, V. S. L. (2020). A política de redução de danos e a aplicabilidade do cuidado em uma unidade de saúde especializada a pessoas que fazem uso abusivo de álcool e outras drogas. Brazilian Journal of health Review, 3(2): 1943-1952.

Ferreira, T. P. S., Sampaio, J., Oliveira, I. L., \& Gomes, L. B. (2019). A família no cuidado em saúde mental: desafios para a produção de vidas. Saúde em Debate, 43(121), 441-449.

Guimarães, L. (2016). Conhecendo um pouco sobre as RAS - Redes de Atenção à Saúde. https://redehumanizasus.net/94734-conhecendo-um-pouco-sobre-asras-redes-de-atencao-a-saude/.

Kantorski, L. P., Cardano, M., Borges, L. R., \& Machado, R. A. (2021). Cotidiano como eixo de intervenção em saúde mental. Escola Anna Nery, 25(1), 1-9.

Kinker, F. S., Moreira, M. I. B., \& Bertuol, C. (2018). O desafio da formação permanente no fortalecimento das Redes de Atenção Psicossocial. Interface (Botucatu), 22(67), 1247-1256.

Melo, E. A., Mendonça, M. H. M., Oliveira, J. R., \& Andrade, G. C. L. (2018). Mudanças na Política Nacional de Atenção Básica: entre retrocessos e desafios. Saúde Debate, 42(1), 38-51.

Nogueira, T. C. T., \& Campos, M. de F. (2016). Trabalho em rede multirprofissional e a interdisciplinaridade: uma proposta na formação de trabalhadores que desenvolvem o cuidado em saúde mental. Cadernos Brasileiros de Saúde Mental/Brazilian Journal of Mental Health, 8 (18).

Monken, M., Peiter, P., Barcellos, C., Rojas, L. I., Navarro, M., Gondim, G. M. M., \& Gracie, R. (2008). O território na saúde: construindo referências para análises em saúde e ambiente. In: Miranda A. C., Barcellos, C., Moreira, J. C., \& Monken, M (orgs.). Território, ambiente e saúde. Rio de Janeiro: Fiocruz.

Pellegrini, A., Filho. (2021). Determinantes sociais. Fundação Oswaldo Cruz. https://pensesus.fiocruz.br/determinantes-sociais.

Santos, A. F., Aguiar, D. M., \& Braga, M. A. P. L. (2020). A efetiva ação de políticas públicas de atendimento aos usuários de drogas como fomentadora da redução de danos. Rev. Juris., 30(1), 89-111.

Santos, J. A. T., \& Oliveira, M. L. F. (2013) Políticas públicas sobre álcool e outras drogas: breve resgate histórico. Saúde \& Transformação Social/ Health \& Social Change, 4(1), 82-89.

Saraceno, B. (1996). Reabilitação psicossocial: uma estratégia para a passagem do milênio. In: Pitta, A. (org). Reabilitação Psicossocial no Brasil. São Paulo: Hucitec.

Saraceno, B. (2001). Libertando identidades: da reabilitação psicossocial à cidadania possível (2a ed.). TeCorá.

Silva, N. dos S., Esperidião, E., Cavalcante, A. C. G., Souza, A. C. S., \& Silva, K. C. C. da. (2013). Desenvolvimento de recursos humanos para atuar nos serviços de saúde mental. Texto Contexto Enfermagem, 22(4), 1142-1151.

Tenório, F. (2002). A reforma psiquiátrica brasileira, da década de 1980 aos dias atuais: história e conceito. História, Ciências, Saúde, 9(1), 25-59.

Yasui, S. (2006). Rupturas e encontros: desafios da Reforma Psiquiátrica Brasileira. Tese de doutorado, Escola Nacional de Saúde Pública da Fundação Oswaldo Cruz, Rio de Janeiro, Brasil. 